\title{
3 Research Square

\section{Transcriptome-Based Identification and Expression Profiling of AP2/ERF Members in Caragana Intermedia and Functional Analysis of CIDREB3}

Kun Liu

Inner Mongolia Agricultural University

Qi Yang

Inner Mongolia Agricultural University

Tianrui Yang

Inner Mongolia Agricultural University

Feiyun Yang

Inner Mongolia Agricultural University

Ruigang Wang

Inner Mongolia Agricultural University

Jingyu Cong

Inner Mongolia Agricultural University

Guojing Li ( $\sim$ liguojing@imau.edu.cn )

Inner Mongolia Agricultuaral University

\section{Research Article}

Keywords: Abiotic stresses, AP2/ERF, Expression profiling, Drought, Caragana intermedia, Transgenic Arabidopsis

Posted Date: August 9th, 2021

DOI: https://doi.org/10.21203/rs.3.rs-734745/v1

License: (c) (1) This work is licensed under a Creative Commons Attribution 4.0 International License. Read Full License

Version of Record: A version of this preprint was published at Molecular Biology Reports on October 22nd, 2021. See the published version at https://doi.org/10.1007/s11033-021-06826-8. 


\section{Abstract}

Caragana. intermedia Kuang et H.C.Fu., a xerophytic deciduous shrub that widely distributed in arid and semi-arid desert areas of North-west China, is highly tolerant to cold, drought, salt-alkali and barren. In this study, the transcriptome $C$. intermedia under drought treatment was screened for the genes encoding the AP2/ERF family of transcription factors. As a result, 22 sequences with complete open reading frames (ORFs) were obtained. All sequences were divided into 13 groups, including the DREB A1 to A6, ERF B1 to B6, and RAV groups. The results of multiple sequence alignment (MSA) analysis, domain distribution analysis, conservative motif analysis and tertiary structure prediction showed that all the AP2/ERF transcription factors contained the AP2 domain. The AP2 domain contained the YRG and RAYD elements. In addition, quantitative real-time polymerase chain reaction (qRT-PCR) was employed to analyze the expression patterns of the 22 AP2/ERF transcription factors in $C$. intermedia. Most of the transcription factors exhibited tissue-specific expression. The expression of most transcription factors was induced to varying degrees by cold, heat, salt and mannitol stress. Furthermore, Heterologous expression of CiDREB3 in transgenic Arabidopsis L. Heynh decreased root length and fresh weight, decreased rosette leaf diameter and number of leaf, and improved drought tolerance during seedlings development. Taken together, the results of the present study help to better understand the functions of the AP2/ERF family of transcription factors in plant responses to multiple abiotic stresses and deeply insight the roles of CiDREB3 in Arabidopsis morphology and conferring abiotic stress tolerance.

\section{Introduction}

Transcription factors play important roles in plant growth, development and response to various environmental stresses. The Apetala2/ethylene response factor (AP2/ERF) transcription factors are a class of important plant transcription factors that are involved in the regulation of plant growth, development and stress response[1]. The AP2/ERF transcription factors contain the conserved AP2 domains consisting of approximately 57 to 70 amino acid residues. The AP2 domain is composed of an amphipathic $\alpha$-helix and three antiparallel $\beta$-sheets. The $\beta$-sheets is the binding region for cis-acting elements[2]. In addition, each AP2 domain contains two conserved elements, YRG and RAYD. The YRG element is located at the N-terminus of the AP2 domain and consists of approximately 19 to 22 amino acid residues. The RAYD element is located at the C-terminus of the AP2 domain and consists of approximately 43 amino acids. These two elements play an important role in the binding of AP2/ERF transcription factors to various cis-elements [3]. For the DREB and ERF transcription factor subfamilies, the $14^{\text {th }}$ and $19^{\text {th }}$ amino acids in the second $\beta$-sheet determine the binding specificity of the transcription factors to various cis-elements $[2,4]$.

Based on sequence similarity among the AP2/ERF-type transcription factors, the number of AP2 domains and the number of other domains, the AP2/ERF transcription factor family in Arabidopsis is divided into 5 subfamilies, namely, the AP2, DREB, ERF, RAV and Soloist subfamilies [4]. The AP2 subfamily contains two AP2 conserved domains, while the RAV subfamily contains one AP2 conserved domain and one B3 conserved domain. The DREB and ERF subfamilies each contain one AP2 conserved domain. Other 
subfamilies also contain one AP2 domain, which, however, does not contain the WLG motif [5]. The DREB and ERF subfamilies are each divided into 6 groups. Specifically, the DREB subfamily is divided into the A1-A6 groups, while the ERF subfamily is divided into the B1 to B6 groups [4]. The nuances in the sequence and structure of the AP2 domain directly affect the specificity of binding to the downstream cisacting elements[6].

To date, a number of plant AP2/ERF transcription factors have been reported. On the basis of the research conducted by Li et al [7], we summarized the species that express the AP2/ERF family of transcription factors that have been reported so far (Table 1). As early as 2002, it was reported by Sakuma et al that there were 145 AP2/ERF transcription factors in A. thaliana. In 2006, Nakano et al further analyzed the AP2/ERF-type transcription factors in $A$. thaliana based on the research conducted by Sakuma et al. It was reported that there were a total of 121 members in the ERF and DREB subfamilies. Specifically, the AP2 subfamily consisted of 17 members, the RAV subfamily consisted of 6 members, and the Soloist subfamily had only 1 member (AL079349). Nakano et al. found that the gene encoding the member of the Soloist subfamily was At4g13040, which was consistent with the results of Sakuma et al. In addition, Nakano et al merged two subfamilies in the Sakuma's classification, DREB and ERF, into one family and named it the ERF family. Eventually, Nakano et al discovered a total of 147 AP2/ERF transcription factors in A. thaliana, including a member of the AP2 subfamily (At5g60120) and a member of the ERF subfamily (At1g22190) that had not been identified by Sakuma et al $[4,5]$.

In 1994, Jofuku et al [8] first discovered that the AP2/ERF transcription factors regulated floral meristem formation, floral organ formation, flower development-related gene expression and seed development in the model plant $A$. thaliana. In 1995, Ohme-Takagi et al [9] isolated certain transcription factors from tobacco and other plants. These transcription factors specifically recognized the GCC-box in the promoter region of ethylene-inducible pathogenesis-related protein genes and participated in the ethylene response process. The transcription factor genes were named EREBP-1, EREBP-2, EREBP-3 and EREBP-4 according to their encoded products. Thereafter, an increasing number of reports were published regarding the function of the AP2/ERF transcription factors. Moreover, the study of the AP2/ERF transcription factors involves an increasingly wide range of species, including model plant such as $A$. thaliana [10], Nicotiana tabacum L. [11] and Glycine max Merr. [12], et al. There are also reports involving other plants such as Triticum aestivum L. [13], Vitis vinifera L.[14] and Malus sieversii (Ledeb.) Roem, et al[15].

A number of reports have demonstrated that the AP2/ERF transcription factors widely participated in the regulation of growth, development and many other biological processes in plants, such as root initiation and formation [16, 17], fruit maturation [18], seed development [8], flower development [19], and somatic embryo development [20]. In addition, the AP2/ERF transcription factors, especially the members of the DREB and ERF subfamilies, are involved in the regulation of responses to abiotic stresses, such as drought, high salt and alkali, high temperature, frost, senescence, oxidation and heavy metal ions, as well as the regulation of responses to biological stresses, such as pathogens and virus infection[1, 21-23]. Furthermore, the AP2/ERF transcription factors play roles in plant hormone-mediated signal transduction 
pathways, including ethylene, abscisic acid (ABA), jasmonic acid, salicylic acid (SA) and gibberellin signaling pathways[1, 21, 24].

Caragana. intermedia Kuang et H.C.Fu. is a xerophytic deciduous shrub belonging to the genus Caragana Fabr. It is mainly distributed in the arid and semi-arid desert areas of Inner Mongolia, Ningxia and northern Shaanxi. Plants of the genus Caragana have the following characteristics: it's cold, drought, saltalkali and barren tolerance. $C$. intermedia has strong vitality and adaptability, and is ideal for soil and water preservation, as a windbreak and for sand fixation. It also can be used as pasture for sheep, camel and cattle. $C$. intermedia is a superior xerophytic shrub species eminently suitable for artificial afforestation in arid desert steppe [25]. However, there are currently few studies focusing on the function of the AP2/ERF-type transcription factors in $C$. intermedia. In this study, genes encoding the drought stress-inducible AP2/ERF transcription factors were screened out from the data set of the $C$. intermedia drought transcriptome and were cloned. Twenty-two sequences with complete open reading frames (ORFs) were obtained and their phylogenetic relationship, physicochemical properties, domains, tertiary structures, conserved motifs and expression patterns in different tissues under various types of stress were analyzed. Then, the morphology and conferring abiotic stress tolerance of CiDREB3 was further studyed in transgenic Arabidopsis. The results of this study provide a basis for exploring the molecular functions of the AP2/ERF transcription factors and the molecular mechanisms of stress tolerance of $C$. intermedia.

\section{Materials And Methods}

\section{Plant sampling and cultivation}

The seeds of $C$. intermedia were collected from Helingeer County, Hohhot, Inner Mongolia Autonomous Region and Siziwangqi, Ulanqab city, Inner Mongolia Autonomous Region, China. The seeds of Arabidopsis thaliana wild-type during this work are Columbia-0 ecotype (Col-0), and mutant of DREB3(dreb3) was obtained from SIGnAL (Salk Institute Genomic Analysis Laboratory) and ABRC (Arabidopsis Biological Resource Center), and the Name/Stock Number was SALK_206788C.

Cultivation of $C$. intermedia: Plump $C$. intermedia seeds free of worm holes were selected, sown in pots containing nutrient-rich soil: vermiculite $(1: 3, \mathrm{v} / \mathrm{v})$, and cultivated in a greenhouse at $25^{\circ} \mathrm{C}$ under long-day conditions ( $16 \mathrm{~h}$ light/8 h dark cycle).

\section{Plant treatments}

The 25-day-old uniformly growing $C$. intermedia seedlings were exposed to 4 different types of stress: cold, heat, salt and mannitol. The treatment procedures were as below: 1) Cold or heat treatment: $C$. intermedia seedlings that were previously grown under normal conditions were transferred to $4{ }^{\circ} \mathrm{C}$ (for cold treatment) or $42{ }^{\circ} \mathrm{C}$ (for heat treatment) in RUMED incubators and cultivated under the $16 \mathrm{~h}$ light/ $8 \mathrm{~h}$ dark condition. 2) Salt or mannitol treatment: At 3 to 4 days after the last watering, $C$.

intermedia seedlings that were previously grown under normal conditions were irrigated with $200 \mathrm{mmol} \mathrm{L}^{-}$ 
${ }^{1} \mathrm{NaCl}$ solution (for salt treatment) or $300 \mathrm{mmol} \mathrm{L}^{-1}$ mannitol solution. The seedlings were exposed to the above stress treatments for 0, 1, 3 and $12 \mathrm{~h}$ respectively. Samples were collected from the aerial parts of the seedlings, rapidly frozen in liquid nitrogen, and stored in a $-80^{\circ} \mathrm{C}$ freezer for subsequent experiments. At each time point, samples were collected from 3 seedlings and mixed together. For each treatment, three independent biological replicates were performed.

Examination of tissue-specific expression: Root, stem and leaf tissues were collected from 25-day-old $C$. intermedia seedlings. Each type of tissue was harvested from 5 seedlings, rapidly frozen in liquid nitrogen, and stored in a $-80^{\circ} \mathrm{C}$ freezer. Three independent biological replicates were performed.

\section{Verification of the coding regions of the AP2/ERF-type genes in $C$. intermedia}

AP2/ERF gene sequences whose expression levels were significantly up-regulated or down-regulated (reads per kilobase per million mapped reads $(\mathrm{RPKM}) \geq 2$ ) were selected from the $C$. intermedia drought transcriptome database (NCBI Accession number: SRP121096) and subjected to sequence alignment analysis using the NCBI Blastn program. Gene sequences containing complete ORFs were verified using specifically designed primers (see supplementary Table 1 for primer sequences). The remaining sequences that lacked a complete ORF were amplified using the rapid amplification of cDNA ends (RACE) technique to obtained the flanking sequences. The flanking sequences were then assembled with the known intermediate fragments using the Vector NTI 10.0 software (Invitrogen), generating gene sequences with complete ORFs. The ORF sequences were verified using specifically designed primers (the primer sequences are summarized in supplementary Table 1). The primers were designed using Primer Premier 5.0 software. Polymerase chain reaction (PCR) amplification was performed using high-fidelity PrimeSTAR HS DNA Polymerase. The complementary DNAs(cDNAs) and the genomic DNAs (gDNAs) of C. intermedia served as PCR templates. The melting temperature (Tm) and extension time varied for different genes in the AP2/ERF family are summarized in Table 2.

\section{Bioinformatic analysis}

The sequences in the drought transcriptome database were subjected to alignment analysis using the NCBI Blastn online alignment tool. The theoretical pI (isoelectric point) and MM (molecular mass) were calculated using the Compute pl/MW online tool (ExPASy, Switzerland). The subcellular localization of proteins was predicted using the WoLF PSORT online program [26]. Protein sequences from different species were aligned using the DNAMAN software.

The conserved protein motifs were analyzed using the MEME online tool [27]. The settings of the parameters were as follows: minimum motif width $=6$; maximum motif width $=50$, maximum number of motifs $=15$. Protein domains were analyzed using the NCBI's Conserved Domain Database (CDD) [28], and the distribution map of the conserved protein domains was plotted using DOG 2.0 software [29]. The tertiary structures of various subfamilies of $C$. intermedia AP2/ERF-type proteins were subjected to homology modeling analysis using SWISS-MODEL, which allowed the establishment of tertiary structure models of AP2/ERF-type proteins in $C$. intermedia. 
The MEGA 6.0 software was used to analyze the homology of the AP2/ERF-type proteins in C. intermedia with those in M. truncatula and $A$. thaliana [30]. The sequences of Medicago-derived AP2/ERF-type proteins were obtained from a study published by Shu et al [31], whereas the sequences of the Arabidopsis-derived AP2/ERF-type proteins were acquired from a study published by Nakano et al [5]. The obtained sequences were compared using ClusterW, and a portion of the sequences that was significantly different in amino acid composition was removed. The algorithm used in the analysis was neighborjoining. The bootstrap value was set to 1000 . The mode used in the analysis was the Poisson model, and the gaps were set to partial deletion.

\section{Real-time fluorescence-based quantitative PCR (qRT-PCR)}

The diluted templates were removed from the $-80^{\circ} \mathrm{C}$ freezer, placed on ice, and thawed naturally. The expression levels of various genes were analyzed at the transcriptional level by real-time fluorescencebased qPCR. The PCR instrument used in the present study was a LightCycler 480 (Roche, Basel, Switzerland), and the fluorescent dye used was SYBR Green Premix II. The CiEF1a of C. intermedia was selected as the internal reference gene (Table S1). Three technical replicates were performed for each sample, while three biological replicates were performed for each gene. The experimental results were analyzed using the $2^{-\Delta \Delta C t}$ method.

\section{Analysis of the expression patterns}

The genes encoding the AP2/ERF family of transcription factors were screened out from the drought transcriptome database of $C$. intermedia. The expression pattern of these genes in various tissues (root, stem, leaf and whole plant) or under different stress conditions (cold, hot, salt and mannitol stress) were analyzed. Briefly, RNA was extracted using the RNAsimple Total RNA Kit (DP419) (Tiangen Biotech (Beijing) Co, Ltd, China) in accordance with the manufacturer's instructions. The first-strand cDNA synthesis was performed using TransScript gDNA Removal and cDNA Synthesis SuperMix (AT311) (Beijing TransGen Biotech Co., Ltd., China) in accordance with the manufacturer's instructions. qRT-PCR was performed using the $C$. intermedia cDNAs that had been diluted 16-fold as templates. The sequences of PCR primers are shown in Table S1. The PCR system and procedure were set up accoding to the manufacturer's instructions. A heat map was constructed using the Heml 1.0 software [32] based on the results of qRT-PCR.

\section{CiDREB3 transgenic Arabidopsis}

To generate the recombinant CiDREB3 overexpression vector, the full-length CDSs of CiDREB3 was amplified using the wild $C$. intermedia cDNA and cloned into the expression vector pCanG-HA using the restriction enzymes Sall/Sacl, under the control of the CaMV35S promoter. The recombinant vectors were expressed in wild-type $A$. thaliana using the floral dipping method, mediated by Agrobacterium tumefaciens (strain GV3101). The empty vector was used as the control.

\section{Morphological observation}




\section{Root length measurement}

For root length measurement under normal condition, wild-type, mutant and transgenic Arabidopsis seedlings germinated for $48 \mathrm{~h}$ were transferred to $1 / 2 \mathrm{MS}$, and hold on upright for 6 days under normal condition. At least 40 seedlings per genotype were calculated for root length measurement. This experiment was repeated three times.

\section{Fresh weight measurement}

The aerial part of transgenic Arabidopsis, wild-type and mutant seedlings grown for 3 weeks under normal condition was chose to measure the fresh weight. it was calculated using 4 plants per parallel group and 3 parallel group per genotype for each experiment. This experiment was repeated three times.

\section{Rosette leaf diameter and leaf number measurement}

The 7th to 9th rosette leaf diameter for three-week-old transgenic Arabidopsis, wild-type and mutant was detected, and using 30 plants per genotype for each experiment. This experiment was repeated three times.

\section{Drought tolerance test}

To evaluate the potential drought tolerance, surface-sterilized seeds from wild-type and CiDREB3 transgenic Arabidopsis were planted on the plate. The plates were incubated at $4^{\circ} \mathrm{C}$ for 3 days in dark before being placed at $22{ }^{\circ} \mathrm{C}$ under 16-h light/8-h dark conditions. Seedlings grown for 3 weeks under a normal watering regime were subjected to drought stress by withholding watering for 14 days and were then re-watered for 2 days. Survival rates and chlorophyll content were calculated and assayed using 28 plants per genotype for each experiment. This experiment was repeated three times.

\section{Results}

\section{Transcriptome-based identification of the AP2/ERF family of genes in $C$. intermedia}

After screening the drought transcriptome database and performing the Blastn sequence alignment analysis, a total of 37 gene sequences with intact AP2 domains were identified, and these included 20 gene sequences containing the complete ORFs. Sequences lacking the complete ORF were amplified using the RACE technique to obtain the flanking sequences. After assembly of the sequences, two more gene sequences with complete ORFs were obtained, namely, CiERF008 (comp92811_c1) and CiERF004 (comp123668_c0). Eventually, 22 sequences with complete ORFs were obtained (Table 3). The 22 gene sequences were compared with the sequences in the TAIR database. The results showed that 3 of the 22 genes belonged to the DREB A1 group, 2 belonged to the DREB A2 group, 2 belonged to the DREB A4 group, 4 belonged to the DREB A5 group, 1 belonged to the DREB A6 group, while 2 belonged to the ERF B1 group, 7 belonged to the ERF B3 group, and 1 belonged to the RAV group. 


\section{Phylogenetic evolution analysis of the AP2/ERF transcription factors in $C$. intermedia}

To investigate the phylogenetic relationship among the $C$. intermedia AP2/ERF transcription factors, MEGA 6.0 software was used to construct a phylogenetic tree from all 22 AP2/ERF transcription factor sequences. The results are shown in Fig. S1. Different background colors represent the different subgroups. The DREB subgroups were clustered in one branch, and those belonging to the ERF B3 subgroup were clustered in the second branch, whereas the 2 members of the ERF B1 subgroup (CIERF004 and CiERFO09) and CiRAV1 were clustered in the third branch.

To further investigate the evolutionary relationship between the AP2/ERF transcription factors in $C$. intermedia and their homologs in other plants, phylogenetic evolution analysis was performed based on the 22 AP2/ERF transcription factor sequences from $C$. intermedia (22), M. truncatula(101) , and $A$. thaliana (128). Proteins from M. truncatula and A. thaliana consisted of DREB-type, ERF-type and RAVtype. None of these sequences belong to the AP2 or Solist subfamilies. As shown in Fig. 1, the sequences were divided into 13 groups, including the DREB A1-A6, ERF B1-B6 and RAV groups. Members of the DREB A1 group were clustered with M. truncatula MtERF021, MtERF023 and MtERF024 (Fig. S2). CiDREB2C and CiDREB2D were clustered with MtERFO48 and AtERFO45 in one branch (Fig. S3). The members of the A4 group of $C$. intermedia were clustered with MtERF025 and MtERF029 (Fig. S4). Some scholars merged all of the above $M$. truncatula sequences into one group, which was named group III. CIERF008, CiRAP2-1, CiERF020 and CiERF017, which are members of the DREB A5 group, were clustered with MtERF018, MtERF014, MtERF017 and MtERF012, respectively (Fig. S4). CiERF061 had a relatively close genetic relationship with AtERF061 (Fig. S5). CiERF004 and CiERF009 were clustered together with MtERF073 (Fig. S6). All members of the ERF B3 group except CiERF109 were derived from the same ancestor (Fig. S7). In addition, the RAV subfamily member CiRAV1 was closely related to MtERF120 and AtRAV2 (Fig. S8).

\section{Analysis of the physicochemical characteristics of the $C$. intermedia AP2/ERF transcription factor family}

The physicochemical properties of the amino acid sequences that were encoded by the 22 genes with complete ORFs were predicted using the online prediction tool Protparam. As shown in Table 3, the number of the amino acids encoded by the 22 genes varied greatly (ranging from 151 to 453 amino acids). The molecular mass of the proteins also varied significantly, ranging from $16.5 \mathrm{kD}$ to $50.9 \mathrm{kD}$. Of the 22 transcription factors, 6 had isoelectric points greater than 7. Among these 6 transcription factors, CiERF009 had the highest isoelectric point (9.51). The other 16 transcription factors had isoelectric points lower than 7, among which, CiDREB2C had the lowest isoelectric point (4.84). Except for CiDREB3 and CiERF020, the other 20 transcription factors were all predicted to locate in the nucleus.

\section{Domains distribution in the AP2/ERF family of transcription factors from $C$. intermedia}

Through protein sequence alignment and analysis, the lengths and positions of the conserved domains in the $22 \mathrm{C}$. intermedia AP2/ERF-type transcription factors were determined. The shortest domain was 57 amino acids in length (CiRAV1), while the longest domain was 70 amino acids (CiDREB2D), which 
conformed to the length of the typical AP2 domain (Table 4). In addition to a conserved AP2 domain, the CiRAV protein contained a B3 domain consisting of 107 amino acids (Fig. 2).

\section{Multiple sequence alignment of the AP2/ERF family of transcription factors from $C$. intermedia}

The AP2 domains of the $22 \mathrm{C}$. intermedia AP2/ERF transcription factors were subjected to multiple sequence alignment using the DNAMAN software. As shown in Fig. 3, all AP2/ERF transcription factors contained the typical elements of the AP2 domain: YRG and RAYD. The YRG element was composed of YRGVRxRxxxGKWVCEVREPNKK, while the RAYD element was composed of RIWLGTFxxxxMAAxAxDVAAxAxRGxxACLNFxxxAxxLxxx. The two elements bind to various cis-acting elements, thereby playing important roles in a variety of signaling pathways. In addition, the YRG element contains $\beta 1$ sheet (VRxR) and $\beta 2$ sheet (KWVCEVRE) structures, while the RAYD element contains a $\beta 3$ sheet (TRIWLGTF) and an amphipathic a-helix (TAEMAARAHDVAALALRG). The above findings indicate that all 22 sequences are typical AP2/ERF transcription factors.

\section{The distribution of the conserved motifs in the AP2/ERF family of transcription factors from $C$. intermedia}

The MEME online tool was used to predict the conserved motifs in the of $22 \mathrm{C}$. intermedia AP2/ERF transcription factors. The results are shown in Fig. 4. All sequences contains the highly conserved motif 1, which contains the classical element of the AP2 domain-RAYD. In addition, 21 of the 22 sequences contains the highly conserved motif 2, which contains the YRG element. The RAYD and YRG elements are

the key components of the AP2 domain. Members of the DREB A1 group contains motif 3. CiDREB1C and CiDREB1F also contain motif 14 . This motif is short in length and contains the conserved sequence $\operatorname{VQQRD}(H) M(Q)$. However, the mechanism of action of motif 14 remains unclear. Members of the DREB A2 group contain motif 5 , motif 12 and motif 13 , among which motif 5 and motif 12 are highly conserved. Two members of the ERF B3 group, CiERF1B and CiERF109, contain motif 15. Motif 15 is short in length and highly conserved.

\section{Prediction of the tertiary structures of the AP2/ERF family of transcription factors from $C$. intermedia}

Protein tertiary structure directly determines protein function. To intuitively understand the tertiary structures of the 22 C. intermedia AP2/ERF transcription factors, in this study, one transcription factor was selected from each subgroup and subjected to homology-based protein tertiary structure modeling. The results are shown in Fig. S9. Since there was little difference in the AP2 domain among the various subgroups, members of the $A 1, A 4, A 5, A 6, B 1$, and $B 3$ groups were modeled using the same template (5wx9.1.A). For the members of the A2 and RAV1 groups, the reference template was 3gcc.1.A. It was predicted that each subgroup contained an amphipathic $\alpha$-helix, as well as $\beta 1, \beta 2$ and $\beta 3$ sheets. These secondary structures are important components of the AP2 domain, among which the a-helix is essential for the stability of plant cell membranes. The $3 \beta$-sheets are arranged in an anti-parallel fashion and located in front of the $a$-helix. The $\beta$-sheet region is responsible for DNA binding. For the DREB and the 
ERF subfamilies, the $14^{\text {th }}$ and $19^{\text {th }}$ amino acids in the second $\beta$-sheet determined the binding specificity of the transcription factors to various cis-acting elements [4].

\section{Tissue-specific expression of the genes encoding AP2/ERF transcription factors in $C$. intermedia}

The tissue-specific expression of the AP2/ERF-type transcription factors in $C$. intermedia were eximined.

The results are shown in Fig. 5. Except for CIDREB3, CITINY2 and CiERF110, the other genes were expressed at relatively low level in the stem. The expression level of these genes were all within two times that of the reference gene. The CIDREB3, CITINY and CiTINY2, which belonged to DREB A4 group, showed relatively high expression level in stem tissue and suggested that these group members might play important roles in stem development. Most of the AP2/ERF-type genes were highly expressed in root, especially for CIRAP2.11, CiERF013-1 and CiERF112, and expression level of CiDREB3 was almost 3 folds in root. In leaves, 6 genes were expressed at relatively high level and showed a fold change greater than 8 , while DREB A4 group, including CiDREB3, showed almost no expression. In addition, CiRAP2.11 was highly expressed in both root and leaves. Tissue-specific expression indicates that these genes might play roles in the development of these tissues. Except for the CiDREB2C gene, members of the DREB A1, DREB A2, DREB A4 and ERF B4 groups were expressed at low level in the leaves. Low level of expression of the CIRAP2.12 and CIERF1A were observed in all tissues examined. The above results demonstrate that the expression of the AP2/ERF family of transcription factors in $C$. intermedia exhibits tissue specificity.

\section{The expression level of the AP2/ERF-type transcription factors encoding genes in $C$. intermedia under various stress treatments}

qRT-PCR was employed to examine the expression levels of 37 genes encoding the AP2/ERF-type transcription factors in $C$. intermedia under various stress treatments. The results are shown in Fig. S10. Overall, the AP2/ERF gene family in $C$. intermedia was inducible by abiotic stresses such as cold, heat, salt and mannitol. Exposure to cold treatment for $12 \mathrm{~h}$ significantly increased the expression levels of most AP2/ERF-type genes. Among these genes, 5 showed a more than 20-fold change in expression level. CIERF017-1 and CIDREB2C had the highest expression levels (fold increase in expression of 68.08 and 57.14 , respectively). Eighteen genes responded rapidly to heat treatment and showed a more than 2-fold change in expression level after exposure to heat treatment for $1 \mathrm{~h}$. Among the 18 genes, CiDREB1C exhibited the most significant response (fold-change in expression of 228). After $12 \mathrm{~h}$ of heat treatment, the expression levels of 7 genes continued to rise. Among these 7 genes, CiDREB1C showed the highest fold increase in expression level (more than 800 folds). In addition, the expression levels of the AP2/ERF family genes showed an incresing trend after $\mathrm{NaCl}$ and mannitol treatment, indicating that these genes might be important in abiotic stress responses.

\section{Heterologous expression of CiDREB3 decreased root length and fresh weight in transgenic Arabidopsis}

According toexpression profiles of AP2/ERF family genes, CiDREB3, which exhibited relatively high expression in stem and root tissue, and low expression in leaf and stress treatments, was selected for 
further analyses. In order to analyze the function of CiDREB3, it was over-expressed in Arabidopsis. Through RT-qPCR analysis in the T3 homozygotes, three independent overexpression lines (CiDREB3-OE8, CiDREB3-OE29 and CiDREB3-OE1) showing relatively high expression level were selected for further study (Fig. S11). CiDREB3 showed the highest homology to AT5G25810.1 andhencethe corresponding mutant, SALK_206788C, was obtained from ABRC (more details in Materials and methods). The transgenic Arabidopsis seedlings germinated for $48 \mathrm{~h}$ were transferred to $1 / 2 \mathrm{MS}$ and hold on for 6 days, then their morphology were observed and compared (Fig. 6). The CiDREB3-overexpressing lines showed significantly shortening root length compared with the wild-type and dreb3 under normal growth conditions. No significant differences in root length were found between the dreb3 mutant and wild-type under normal growth conditions (Fig. 6c).

Then, we measured the fresh weight of the three-week-old plants, the OE8 and OE29 lines showed significantly lower fresh weight than wild-type, while OE1 line showed a similar fresh weight as the wildtype. The reason may be the expression level of CiDREB3 in OE8 and OE29 lines were higher thanthat in OE1 line, which has a dose effect on transgenic Arabidopsis. By contrast, dreb3 exhibited a significantly higher fresh weight than wild-type (Fig. 6b). These results suggested that overexpression of CiDREB3 could cause a dwarf phenotype in transgenic Arabidopsis.

\section{Heterologous expression of CiDREB3 decreased rosette leaf diameter and number of leaf in transgenic Arabidopsis}

We observed the morphological differences of three-week-old transgenic Arabidopsis under normal growth condition, and the growth of OE8 line was significantly delayed and the leaves were smaller and curled up compared with wild-type. While dreb3 line grow faster and larger than the wild-type (Fig. 7a). The rosette leaves diameter of OE8 was also significantly lower than that of the wild-type and dreb3. The dreb3 showed significantly longer rosette leaves diameter than wild-type (Fig. 7b). In addition, the number of leaf in OE8 line was obviously less than that in other lines (Fig. 7c). These results suggested that overexpression of CiDREB3 could cause a dwarf, delayed and poor growth phenotype in transgenic Arabidopsis.

\section{Heterologous expression of CiDREB3 improved drought tolerance in transgenic Arabidopsis}

Three-week-old seedlings were exposed to drought stress by withholding watering for 14 days and were then rewatered for 2 days. The CiDREB3 overexpression lines were insensitive to drought, had significantly increased survival rates and less wilting and yellowing compared with wild-type (Fig. 8a and 8b). In addition, OE8 and OE29 lines showed a higher chlorophyll level than the wild-type under drought stress. After re-watering for 2 days, the majority of wild-type plants never recovered, while the overexpression lines exhibited a significantly higher survival ratio (Fig. 8c). These results confirmed that CiDREB3 enhanced the tolerance of Arabidopsis to drought stress, and CiDREB3 acted as a positive regulator in the plant response to drought. 


\section{Discussion}

\section{The differences between the AP2/ERF-type transcription factors of the same species}

Different scholars have obtained different statistical results when investigating the AP2/ERF transcription factor genes from the same species. For example, Nakano et al reported that there were a total of 157 AP2/ERF transcription factor genes in 0 . sativa [5], while Sharnoi et al reported 163 AP2/ERF transcription factor genes [33]. Chen et al reported that $B$. distachyon had 149 genes of the AP2/ERF transcription factor family, which could be divided into four subfamilies [34]. Cui et al also carried out statistical analysis on the AP2/ERF transcription factor genes from B. distachyon. A total of 141 genes of the AP2/ERF transcription factor family were identified, which were also divided into four subfamilies [35]. Wu et al reported that Phyllostachys edulis (Carrière) J. Houz. had a total of 121 AP2/ERF transcription factors [36]. Later, Huang et al reported that there were a total of 142 AP2/ERF transcription factors in P. edulis [37]. Song et al reported that there were a total of 291 AP2/ERF transcription factors in B. rapa [38]. In the same year, Liu et al found 281 members of the AP2/ERF transcription factor family in B. rapa [39]. We have summarized the reasons behind such phenomenon, and the possible reasons are as follows: (1) Certain genes in the AP2/ERF family undergo alternative splicing. Some scholars believe that each splice variant is a gene, while others believe that all splice variants derived from the same gene should be counted as one gene. As a result, there was a discrepancy in the number of AP2/ERF genes [35, 40]. (2) Some scholars believe that all genes containing the AP2 domain belong to the AP2/ERF transcription factor family, while other scholars do not count the genes that contain other domains besides the AP2 domain as members of the AP2/ERF transcription factor family, which results in a different number of AP2/ERF genes [38, 39]. (3) When analyzing the AP2/ERF gene family in $P$. edulis, some scholars have included genes with an AP2/ERF domain integrity of less than $70 \%$ in the AP2/ERF family [37]. However, other scholars did not include such genes and only counted the genes with intact AP2/ERF domains. Again, this caused a discrepancy in the number of AP2/ERF genes [36].

\section{Analysis of the AP2/ERF transcription factor family in leguminous plants}

Currently, genome-wide identification and analysis of the AP2/ERF transcription factor family has been performed in the sequenced leguminous plants. For example, Zhang et al identified 148 members of the AP2/ERF transcription factor family in tetraploid G. max [41]. Genes encoding one AP2 domain were classified into one group, which was named the ERF family. The ERF family consists of 120 genes. Among the 120 genes, 98 genes that were capable of encoding one complete AP2 domain were called ERF family genes. The 98 ERF family genes (GMERF001 to GmERF098) were further divided into the DREB subfamily (36 genes) and the ERF subfamily (62 genes). The remaining 22 genes were unable to encode the complete AP2 domain, and the authors did not further group the 22 genes. Agarwal et al counted the number of AP2/ERF transcription factors in 5 types of sequenced leguminous plants [42] (Table 1). The results showed that there were 147 AP2/ERF-type transcription factors in C. arietinum, 176 in Cajanus cajan (L.) Millsp., 131 in M. truncatula, 179 in Phaseolus vulgaris Linn., and 140 in L. 
corniculatus. Shu et al analyzed the M. truncatula AP2/ERF transcription factor family at the genomic level and obtained somewhat different results. In M. truncatula, Shu et al. identified a total of 123 AP2/ERF transcription factors and reclassified and named these transcription factors [31].

\section{Analysis of the expression patterns of the AP2/ERF-type transcription factors in C. intermedia}

The AP2/ERF transcription factor genes display tissue-specific expression in many plants. There is a total of 291 AP2/ERF transcription factor genes in B. rapa. Among the 291 genes, 31.95\% are specifically expressed in the roots, $22.9 \%$ are specifically expressed in the seeds, and $20.06 \%$ are highly expressed in the leaves. In contrast, only a small number of genes are specifically expressed in flowers, pods and buds [38]. In this study, qRT-PCR was performed to examine the expression levels of the 37 AP2/ERF transcription factors in different tissues of $C$. intermedia (Fig. 5). It was found that the expression levels of these genes varied greatly in the roots, stems and leaves and displayed a certain degree of tissue specificity. The CIDREB3 showed relatively high expression level in stem and root tissue, suggested that these subgroup members might play important roles in stem and root development.

In addition, many studies show that all AP2/ERF-type transcription factors are inducible by a variety of abiotic stresses. For example, among the 106 AP2/ERF transcription factors examined in Brachypodium distachyon (L.)., 69 were significantly induced by cold stress and 16 were induced by drought stress [35]. The expression levels of these transcription factors were increased by more than 2 folds after exposure to stress. In 0 . sativa, 70 genes in the AP2/ERF transcription factor family are inducible by cold, drought, flooding, osmotic, salt and hormonal stress [33]. In this study, qRT-PCR was employed to examine the expression patterns of 37 AP2/ERF transcription factor genes under various stresses (Fig. S10). The results showed that these genes were all inducible by cold, heat, $\mathrm{NaCl}$ and mannitol. After cold treatment, 17 genes showed a more than 2-fold increase in expression level, while 8 genes showed a more than 10fold increase in expression level. After heat treatment, the expressions levels of 18 genes showed a more than 2-fold increase. After $\mathrm{NaCl}$ treatment, 14 genes showed a more than 2-fold increase in expression level. After mannitol treatment, there were 12 genes with a more than 2-fold increase in expression. Interestingly, CiDREB3 was induced negatively by cold and heat stress. The expression level of CiDREB3 showed a trend of decreasing first and then increasing in salt stress, while it was not induced by mannitol, and these results were accordant with that in $C$. intermedia drought transcriptome database. In A. thaliana, AtTINY2 was inducible by SA, ABA, mechanical damage, cold, drought and $\mathrm{NaCl}$ [43]. Another gene that belonged to the same group (the A4 group) as AtTINY2 and showed a high sequence similarity was AtTINY. Studies have shown that AtTINY is induced by drought, cold, ethylene and methyl jasmonate. The above results demonstrated that the AP2/ERF transcription factors are inducible by a variety of abiotic stresses.

\section{Phenotype analysis of CiDREB3 in transgenic Arabidopsis}

A phylogenetic tree was constructed from the $22 \mathrm{C}$. intermedia AP2/ERF transcription factor sequences with complete ORFs, $101 \mathrm{M}$. truncatula AP2/ERF sequences and $128 \mathrm{~A}$. thaliana AP2/ERF sequences (Fig. 1). The higher the similarity between genes, the closer their functions are. Among the $A$. thaliana 
genes, AtERF041 (AtTINY2) showed the highest similarity to CiDREB3. It has been reported that overexpression of AtTINY causes dwarfism and severely delayed growth and development in plants [44]. In addition, AtTINY participates in the ethylene-responsive signaling pathways and induces apparent triple response in the absence of ethylene (shortening and thickening of the hypocotyls, shortening of the roots, and exaggeration of the curvature of the apical hook) [45]. The expression levels of the stressrelated genes such as COR6.6, COR15A and COR78 were upregulated in AtTINY transgenic A. thaliana [44]. Our results showed that overexpression of CiDREB3 in Arabidopsis decreased root length and fresh weight, decreased rosette leaf diameter and number of leaf (Fig. 6; Fig. 7), and these results are consistance with the function of AtTINY2. The dwarfism and severely delayed growth phenotype in CiDREB3 overexpression lines was possibly due to the high expression level in stem and root tissue, and CiDREB3 was a negatively regulator in growth and development and negatively regulates stem and root growth in Arabidopsis thaliana.

What's more, transgenic Arabidopsis lines overexpressing CiDREB3 improved drought tolerance during seedlings development (Fig. 8), and the result is similar to StDREB1, also belongs to DREB A4 group, in potato. Drought and salt tolerance were increased by homologous expression of StDREB1 in potato during seedlings growth, and free proline content and expression level of P5CS1 were significantly higher than those in Col-0 under salt stress. Due to CiDREB3 could stunt plant growth and have a negatively impact on plant growth, it is deduced that the CiDREB3 participated in regulating resopnse to drought stress through slowing plant growth and development.

In summary, 22 genes with complete ORFs were identified and divided into 13 groups, multiple sequence alignment (MSA), domain distribution, conservative motif, tertiary structure prediction of these genes were systematically analyzed and verified that these genes were typical AP2/ERF transcription factors. What's more, the expression profile of AP2/ERF transcription factors in $C$. intermedia were tissue-specific and involved in different stress such as cold, heat, salt and mannitol. Additionally, overexpression of CiDREB3 in A. thaliana resulted in dwarfism and severely delayed growth and development, and drought tolerance phenotype in plants compared with the wild-type. This study will provide a further understanding of AP2/ERF transcription factor in plant development and stress resistance, and will provide the basis for future functional studies on AP2/ERFs.

\section{Declarations}

Acknowledgements This work was supported by grant from National Natural Science Foundation of China (Grant numbers: 31560199).

Author contributions Guojing Li and Qi Yang conceived and designed the research. Kun Liu and Tianrui Yang participated in the experiment and data collection together. Qi Yang and Kun Liu contributed new reagents or analytical tools. Kun Liu, Qi Yang and Feiyun Yang analyzed the data. Kun Liu wrote first draft of the manuscript. Jingyu Cong and Ruigang Wang guided the writing and modification of the paper. All authors read and approved the final manuscript. 
Conflict of interest The authors declare that there is no conflict of interest.

Ethical approval This manuscript does not contain any studies conducted on human or animal subjects.

Consent to participate All authors listed in the article have approved the manuscript that is enclosed.

Consent to publish The manuscript is approved by all authors for publication.

Informed consent All authors agree and give consent for the publication.

\section{References}

1. Licausi F, Ohme-Takagi M, Perata P (2019) APETALA2/Ethylene Responsive Factor (AP2/ERF) transcription factors: mediators of stress responses and developmental programs. The New phytologist 199(3):639-649. https://doi.org/10.1111/nph.12291

2. Allen MD, Yamasaki K, Ohme-Takagi M, Tateno M, Suzuki M (1998) A novel mode of DNA recognition by a beta-sheet revealed by the solution structure of the GCC-box binding domain in complex with DNA. The EMBO journal 17(18):5484-5496. https://doi.org/10.1093/emboj/ 17.18.5484

3. Okamuro JK, Caster B, Villarroel R, Van Montagu M, Jofuku KD (1997) The AP2 domain of APETALA2 defines a large new family of DNA binding proteins in Arabidopsis. Proceedings of the National Academy of Sciences of the United States of America 94(13):7076-7081. https://doi.org/10.1073/pnas.94.13.7076

4. Sakuma Y, Liu Q, Dubouzet JG, Abe H, Shinozaki K, Yamaguchi-Shinozaki K (2002) DNA-binding specificity of the ERF/AP2 domain of Arabidopsis DREBs, transcription factors involved in dehydrationand cold-inducible gene expression. Biochemical and biophysical research communications 290(3):9981009. https://doi.org/10.1006/bbrc.2001. 6299

5. Nakano T, Suzuki K, Fujimura T, Shinshi H (2006) Genome-wide analysis of the ERF gene family in Arabidopsis and rice. Plant physiology 140(2):411-432. https://doi.org/10.1104/pp.105.073783

6. Xue GP (2002) Characterisation of the DNA-binding profile of barley HvCBF1 using an enzymatic method for rapid, quantitative and high-throughput analysis of the DNA-binding activity. Nucleic acids research 30(15): e77. https://doi.org/10.1093/nar/gnf076

7. Li MY, Xu ZS, Huang Y, Tian C, Wang F, Xiong AS (2015) Genome-wide analysis of AP2/ERF transcription factors in carrot (Daucus carota L.) reveals evolution and expression profiles under abiotic stress. Molecular genetics and genomics 290(6):2049-2061. https://doi.org/10.1007/ s00438-015-1061-3

8. Jofuku KD, den Boer BG, Van Montagu M, Okamuro JK (1994) Control of Arabidopsis flower and seed development by the homeotic gene APETALA2. The Plant cell 6(9):1211-1225. https:// doi.org/10.1105/tpc.6.9.1211 
9. Ohme-Takagi M, Shinshi H (1995) Ethylene-inducible DNA binding proteins that interact with an ethylene-responsive element. The Plant cell 7(2):173-182. https://doi.org/10.1105/tpc.7.2.173

10. Scarpeci TE, Frea VS, Zanor MI, Valle EM (2017) Overexpression of AtERF019 delays plant growth and senescence, and improves drought tolerance in Arabidopsis. Journal of experimental botany 68(3):673685. https://doi.org/10.1093/jxb/erw429

11. Wen W, Xie Z, Yu G, Zhao C, Zhang J, Huang L, Xu B, Huang B (2018) Switchgrass PvDREB1C plays opposite roles in plant cold and salt tolerance in transgenic tobacco. Hereditas 155:15. https:// doi.org/10.1186/s41065-017-0050-4

12. Yu Y, Duan X, Ding X, Chen C, Zhu D, Yin K, Cao L, Song X, Zhu P, Li Q, NIsaZU, Yu J, Du J, Song Y, Li H, Liu B, Zhu Y (2017)A novel AP2/ERF family transcription factor from Glycine soja, GsERF71, is a DNA binding protein that positively regulates alkaline stress tolerance in Arabidopsis. Plant molecular biology 94(4-5):509-530. https://doi.org/10.1007/s11103-017-0623-7

13. Kulkarni M, Soolanayakanahally R, Ogawa S, Uga Y, Selvaraj MG, Kagale S (2017) Drought Response in Wheat: Key Genes and Regulatory Mechanisms Controlling Root System Architecture and Transpiration Efficiency. Frontiers in chemistry 5:106. https://doi.org/10.3389/ fchem. 2017.00106

14. Wu J, Folta KM, Xie Y, Jiang W, Lu J, Zhang Y (2017) Overexpression of Muscadinia rotundifolia CBF2 gene enhances biotic and abiotic stress tolerance in Arabidopsis. Protoplasma 254(1):239-251. https://doi.org/10.1007/s00709-015-0939-6

15. Liao X, Guo X, Wang Q, Wang Y, Zhao D, Yao L, Wang S, Liu G, Li T (2017) Overexpression of MsDREB6.2 results in cytokinin-deficient developmental phenotypes and enhances drought tolerance in transgenic apple plants. The Plant journal: for cell and molecular biology 89(3):510526. https://doi.org/10.1111/tpj.13401

16. Imin N, Nizamidin M, Wu T, Rolfe BG: Factors involved in root formation in Medicago truncatula. Journal of experimental botany2007, 58(3):439-451. https://doi.org/10.1093/jxb/erl224

17. Kitomi Y, Ito H, Hobo T, Aya K, Kitano H, Inukai Y (2011) The auxin responsive AP2/ERF transcription factor CROWN ROOTLESS5 is involved in crown root initiation in rice through the induction of OsRR1, a type-A response regulator of cytokinin signaling. The Plant journal: for cell and molecular biology 67(3):472-484. https://doi.org/10.1111/j.1365-313X.2011.04610.x

18. Chung MY, Vrebalov J, Alba R, Lee J, McQuinn R, Chung JD, Klein P, Giovannoni J (2010) A tomato (Solanum lycopersicum) APETALA2/ERF gene, SIAP2a, is a negative regulator of fruit ripening. The Plant journal: for cell and molecular biology 64(6):936-947. https://doi.org/10.1111/j.1365-313X.2010.04384.x

19. Maes T, Van de Steene N, Zethof J, Karimi M, D'Hauw M, Mares G, Van Montagu M, Gerats T (2001) Petunia Ap2-like genes and their role in flower and seed development. The Plant cell 13(2):229-244. 
20. Elliott RC, Betzner AS, Huttner E, Oakes MP, Tucker WQ, Gerentes D, Perez P, Smyth DR (1996) AINTEGUMENTA, an APETALA2-like gene of Arabidopsis with pleiotropic roles in ovule development and floral organ growth. The Plant cell 8(2):155-168. https://doi.org/ 10.1105/ tpc.8.2.155

21. Feng K, Hou XL, Xing GM, Liu JX, Duan AQ, Xu ZS, Li MY, Zhuang J, Xiong AS (2020) Advances in AP2/ERF super-family transcription factors in plant. Critical reviews in biotechnology 40(6):750-776. https://doi.org/10.1080/07388551.2020.1768509

22. Liu W, Li Q, Wang Y, Wu T, Yang Y, Zhang X, Han Z, Xu X (2017) Ethylene response factor AtERF72 negatively regulates Arabidopsis thaliana response to iron deficiency. Biochemical and biophysical research communications 491(3):862-868. https://doi.org/10.1016/j.bbrc.2017.04.014

23. Jin R, Kim BH, Ji CY, Kim HS, Li HM, Ma DF, Kwak SS (2017) Overexpressing IbCBF3 increases low temperature and drought stress tolerance in transgenic sweetpotato. Plant physiology and biochemistry 118:45-54. https://doi.org/10.1016/j.plaphy. 2017.06.002

24. Caarls L, Van der Does D, Hickman R, Jansen W, Verk MC, Proietti S, Lorenzo O, Solano R, Pieterse CM, Van Wees SC (2017) Assessing the Role of ETHYLENE RESPONSE FACTOR Transcriptional Repressors in Salicylic Acid-Mediated Suppression of Jasmonic Acid-Responsive Genes. Plant \& cell physiology 58(2):266-278. https://doi.org/10.1093/pcp/pcw187

25. Han X, Feng Z, Xing D, Yang Q, Wang R, Qi L, Li G (2015) Two NAC transcription factors from Caragana intermedia altered salt tolerance of the transgenic Arabidopsis. BMC plant biology 15:208. https://doi.org/10.1186/s12870-015-0591-5

26. Nakai K, Horton P (1999) PSORT: a program for detecting sorting signals in proteins and predicting their subcellular localization. Trends in biochemical sciences 24(1):34-36. https://doi.org/ $10.1016 / \mathrm{s} 0968-0004(98) 01336-x$

27. Bailey TL, Johnson J, Grant CE, Noble WS (2015) The MEME Suite. Nucleic acids research 43(W1): W39-49. https://doi.org/10.1093/nar/gkv416

28. Marchler-Bauer A, Bo Y, Han L, He J, Lanczycki CJ, Lu S, Chitsaz F, Derbyshire MK, Geer RC, Gonzales NR, Gwadz M, Hurwizt D, Lu F, Marchler GH, Song JS, Thanki N, Wang Z, Yamashita RA, Zhang D, Zheng C, Geer LY, Bryant SH (2017)CDD/SPARCLE: functional classification of proteins via subfamily domain architectures. Nucleic acids research 45(D1): D200-D203. https://doi.org/ 10.1093/nar/gkw1129

29. Ren J, Wen L, Gao X, Jin C, Xue Y, Yao X (2009) DOG 1.0: illustrator of protein domain structures. Cell research 19(2):271-273. https://doi.org/10.1038/cr.2009.6

30. Tamura K, Stecher G, Peterson D, Filipski A, Kumar S (2013) MEGA6: Molecular Evolutionary Genetics Analysis version 6.0. Molecular biology and evolution 30(12):2725-2729. https://doi.org/ 
31. Shu Y, Liu Y, Zhang J, Song L, Guo C (2015) Genome-Wide Analysis of the AP2/ERF Superfamily Genes and their Responses to Abiotic Stress in Medicago truncatula. Frontiers in plant science 6:1247. https://doi.org/10.3389/fpls.2015.01247

32. Deng W, Wang Y, Liu Z, Cheng H, Xue Y (2014) Heml: a toolkit for illustrating heatmaps. PloS one 9(11): e111988. https://doi.org/10.1371/journal.pone.0111988

33. Sharoni AM, Nuruzzaman M, Satoh K, Shimizu T, Kondoh H, Sasaya T, Choi IR, Omura T, Kikuchi S (2011) Gene structures, classification and expression models of the AP2/EREBP transcription factor family in rice. Plant \& cell physiology 52(2):344-360. https://doi.org/10.1093/pcp/pcq196

34. Chen L, Han J, Deng X, Tan S, Li L, Li L, Zhou J, Peng H, Yang G, He G, Zhang W (2016) Expansion and stress responses of AP2/EREBP superfamily in Brachypodium distachyon. Scientific reports 6:21623. https://doi.org/10.1038/srep21623

35. Cui L, Feng K, Wang M, Wang M, Deng P, Song W, Nie X (2016) Genome-wide identification, phylogeny and expression analysis of AP2/ERF transcription factors family in Brachypodium distachyon. BMC genomics 17(1):636. https://doi.org/10.1186/s12864-016-2968-8

36. Wu H, Lv H, Li L, Liu J, Mu S, Li X, Gao J (2015) Genome-Wide Analysis of the AP2/ERF Transcription Factors Family and the Expression Patterns of DREB Genes in Moso Bamboo (Phyllostachys edulis). PloS one 10(5): e0126657. https://doi.org/10.1371/journal.pone.0126657

37. Huang Z, Zhong XJ, He J, Jiang MY, Yu XF, Li X (2016) [Identification and characterization of AP2/ERF transcription factors in moso bamboo (Phyllostachys edulis)]. Molekuliarnaia biologiia 50(5):785-796. https://doi.org/10.7868/S0026898416050062

38. Song X, Li Y, Hou X (2013) Genome-wide analysis of the AP2/ERF transcription factor superfamily in Chinese cabbage (Brassica rapa ssp. pekinensis). BMC genomics 14:573. https://doi.org/ 10.1186/14712164-14-573

39. Liu Z, Kong L, Zhang M, Lv Y, Liu Y, Zou M, Lu G, Cao J, Yu X (2013) Genome-wide identification, phylogeny, evolution and expression patterns of AP2/ERF genes and cytokinin response factors in Brassica rapa ssp. pekinensis. PloS one 8(12): e83444. https://doi.org/10.1371/journal.pone. 0083444

40. Mochida K, Yoshida T, Sakurai T, Yamaguchi-Shinozaki K, Shinozaki K, Tran LS (2011) In silico analysis of transcription factor repertoires and prediction of stress-responsive transcription factors from six major gramineae plants. DNA research: an international journal for rapid publication of reports on genes and genomes 18(5):321-332. https://doi.org/10.1093/ dnares/ dsr019

41. Zhang G, Chen M, Chen X, Xu Z, Guan S, Li LC, Li A, Guo J, Mao L, Ma Y (2008) Phylogeny, gene structures, and expression patterns of the ERF gene family in soybean (Glycine max L.). Journal of 
42. Agarwal G, Garg V, Kudapa H, Doddamani D, Pazhamala LT, Khan AW, Thudi M, Lee SH, Varshney RK (2016) Genome-wide dissection of AP2/ERF and HSP90 gene families in five legumes and expression profiles in chickpea and pigeonpea. Plant biotechnology journal 14(7):1563-1577.

https://doi.org/10.1111/pbi.12520

43. Wei G, Pan Y, Lei J, Zhu YX (2005) Molecular cloning, phylogenetic analysis, expressional profiling and in vitro studies of TINY2 from Arabidopsis thaliana. Journal of biochemistry and molecular biology 38(4):440-446. https://doi.org/10.5483/bmbrep.2005.38.4.440

44. Sun S, Yu JP, Chen F, Zhao TJ, Fang XH, Li YQ, Sui SF (2008) TINY, a dehydration-responsive element (DRE)-binding protein-like transcription factor connecting the DRE- and ethylene-responsive elementmediated signaling pathways in Arabidopsis. The Journal of biological chemistry 283(10):6261-6271. https://doi.org/10.1074/jbc. M706800200

45. Wilson K, Long D, Swinburne J, Coupland G (1996) A Dissociation insertion causes a semidominant mutation that increases expression of TINY, an Arabidopsis gene related to APETALA2. The Plantcell 8(4):659-671. https://doi.org/10.1105/tpc.8.4.659

\section{Tables}

Due to technical limitations, table 1-4 is only available as a download in the Supplemental Files section.

\section{Figures}


Fig. 1

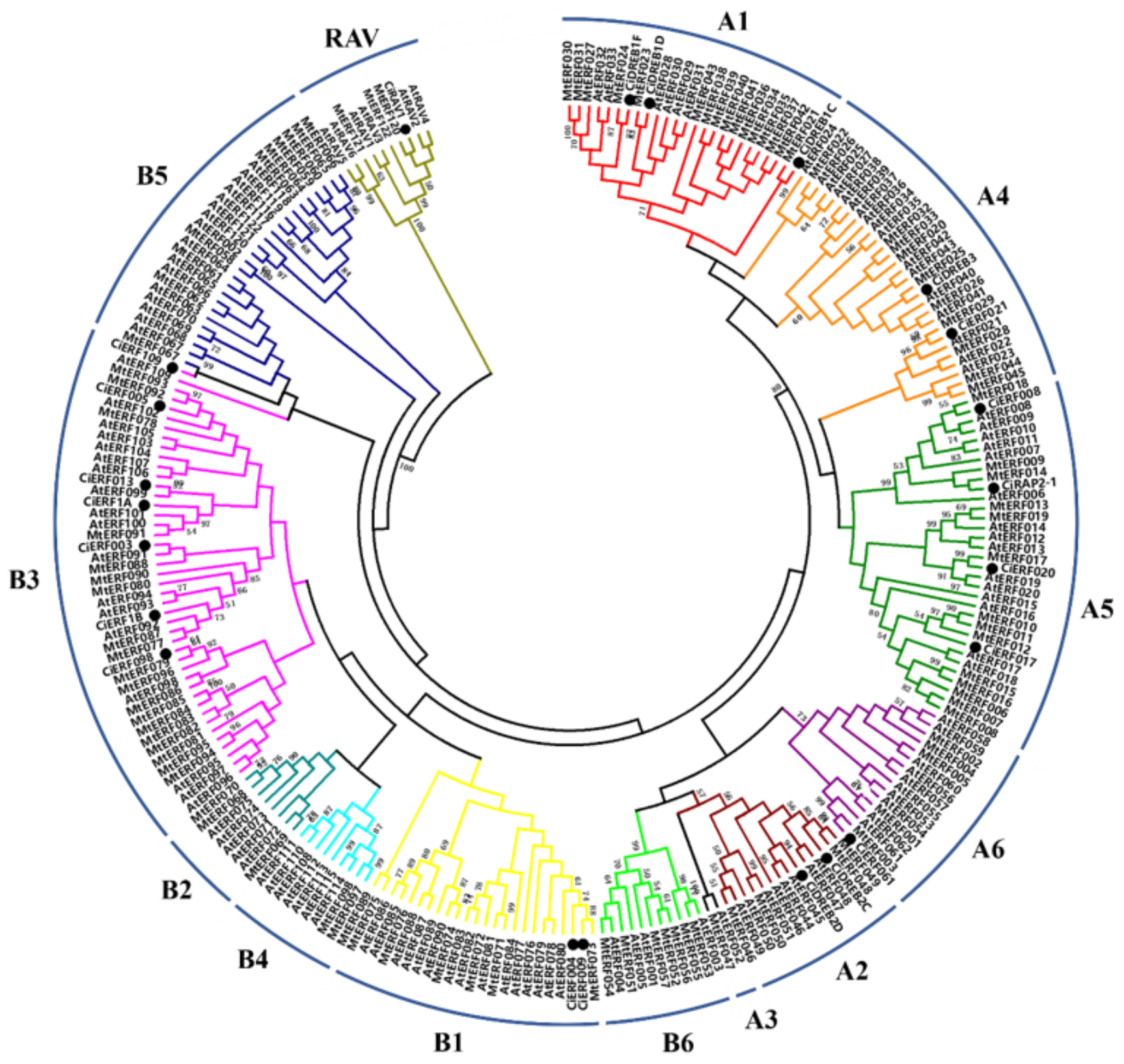

Figure 1

Phylogenetic evolution analysis of the AP2/ERF family of transcription factors from Caragana intermedia, Medicago truncatula and Arabidopsis thaliana A1 to A6: DREB subfamily; B1 to B3: ERF subfamily; RAV: RAV subfamily. The black circles represent the proteins from C. intermedia. 
Fig. 2

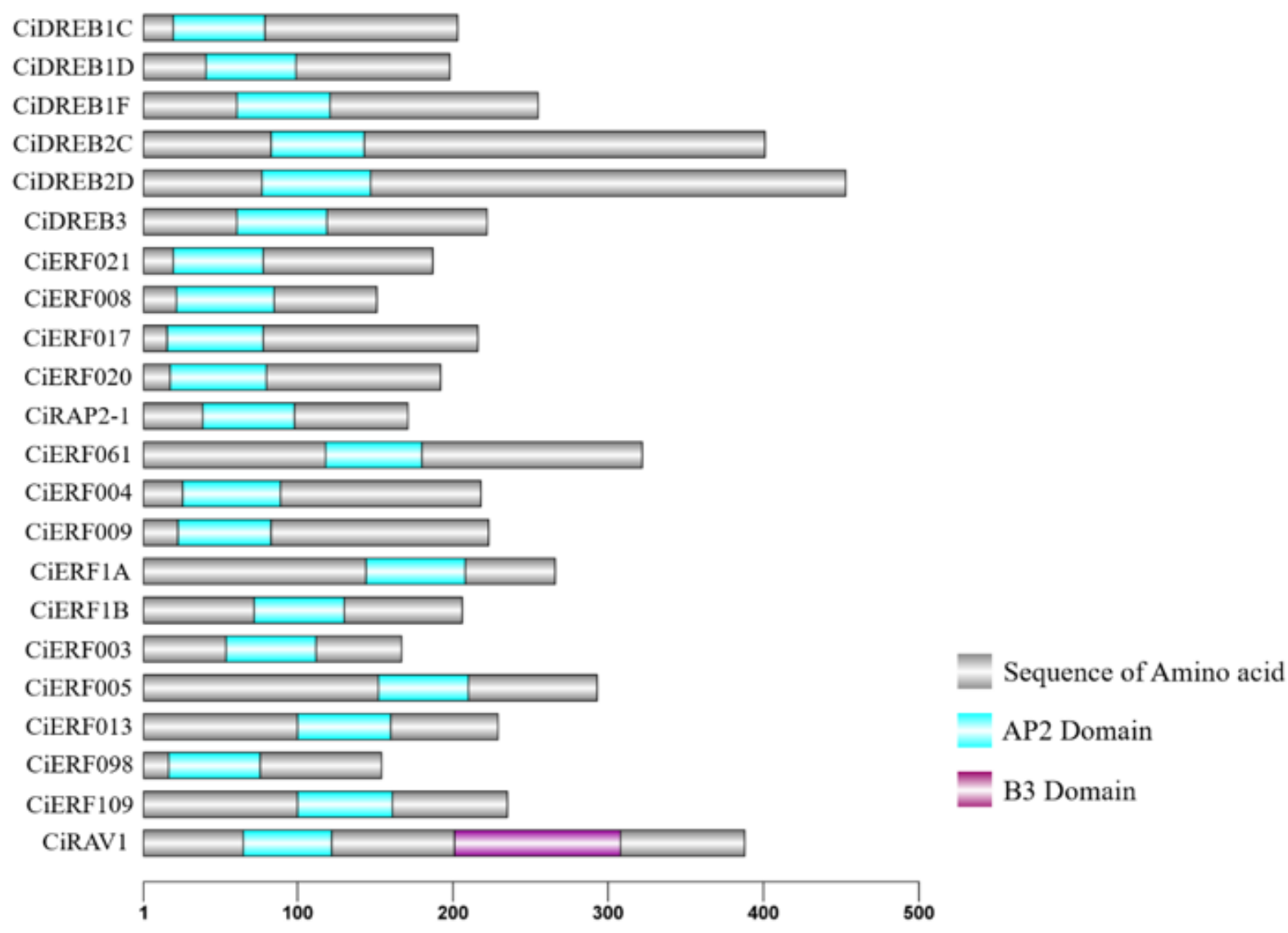

Figure 2

Analysis of the conserved domains in the AP2/ERF family of transcription factors from C. intermedia 
Fig. 3

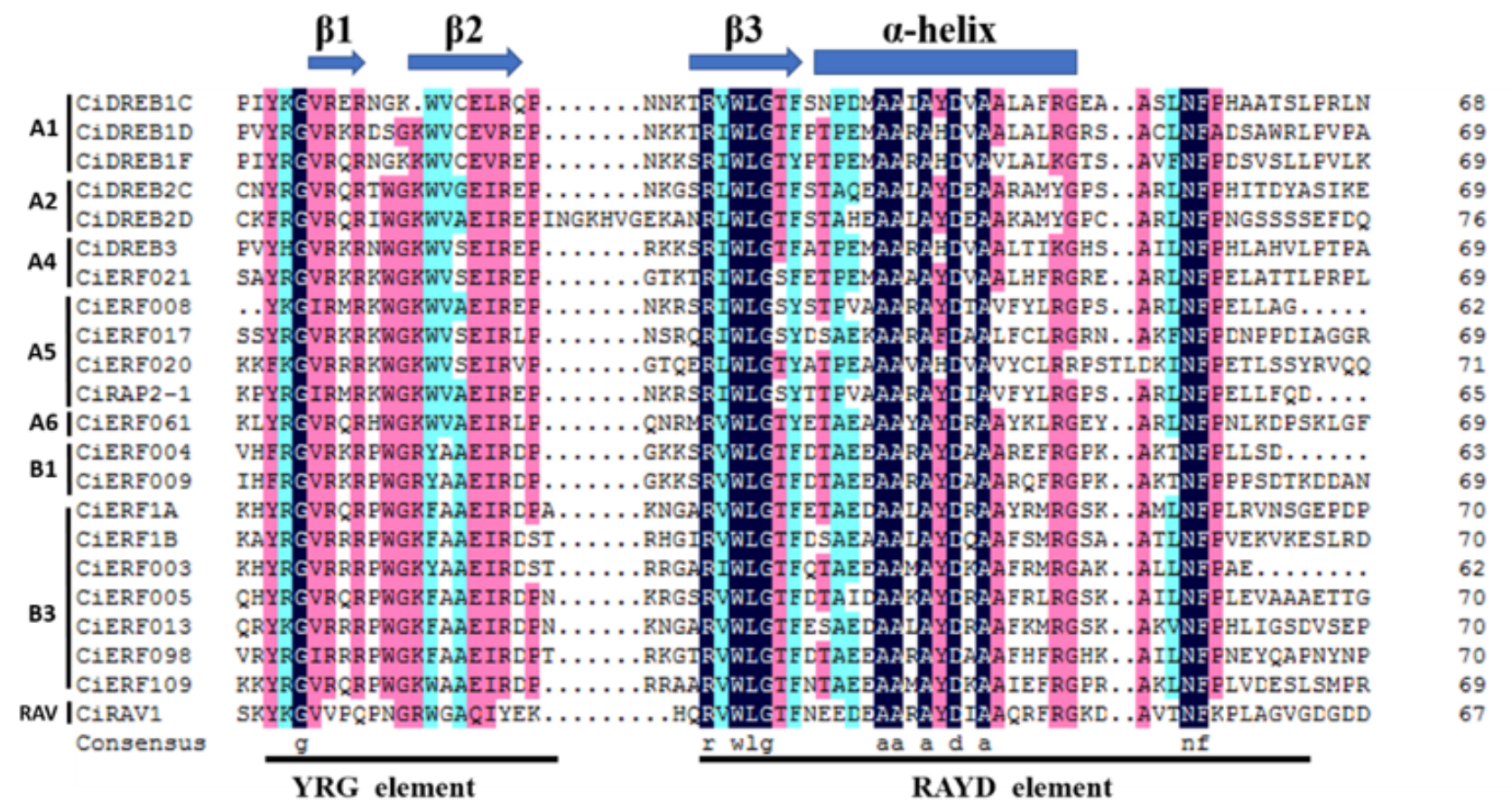

\section{Figure 3}

Multiple sequence alignment of the conserved domains of the AP2/ERF transcription factor family in C. intermedia A1 to A6: DREB subfamily; B1 and B3: ERF subfamily; RAV: RAV subfamily $\beta 1, \beta 2, \beta 3$ sheets and a-helix: the secondary structure of amino acids; YRG and RAYD indicate these elements 
Fig. 4

a

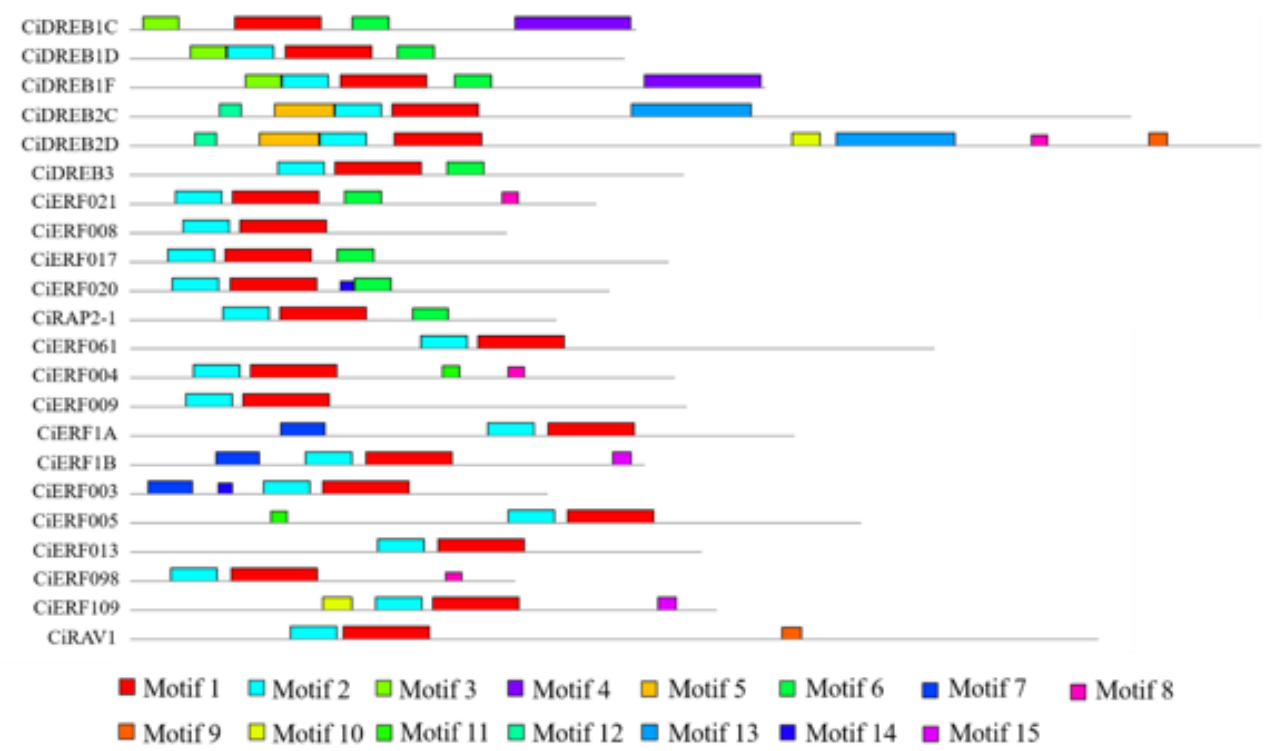

b

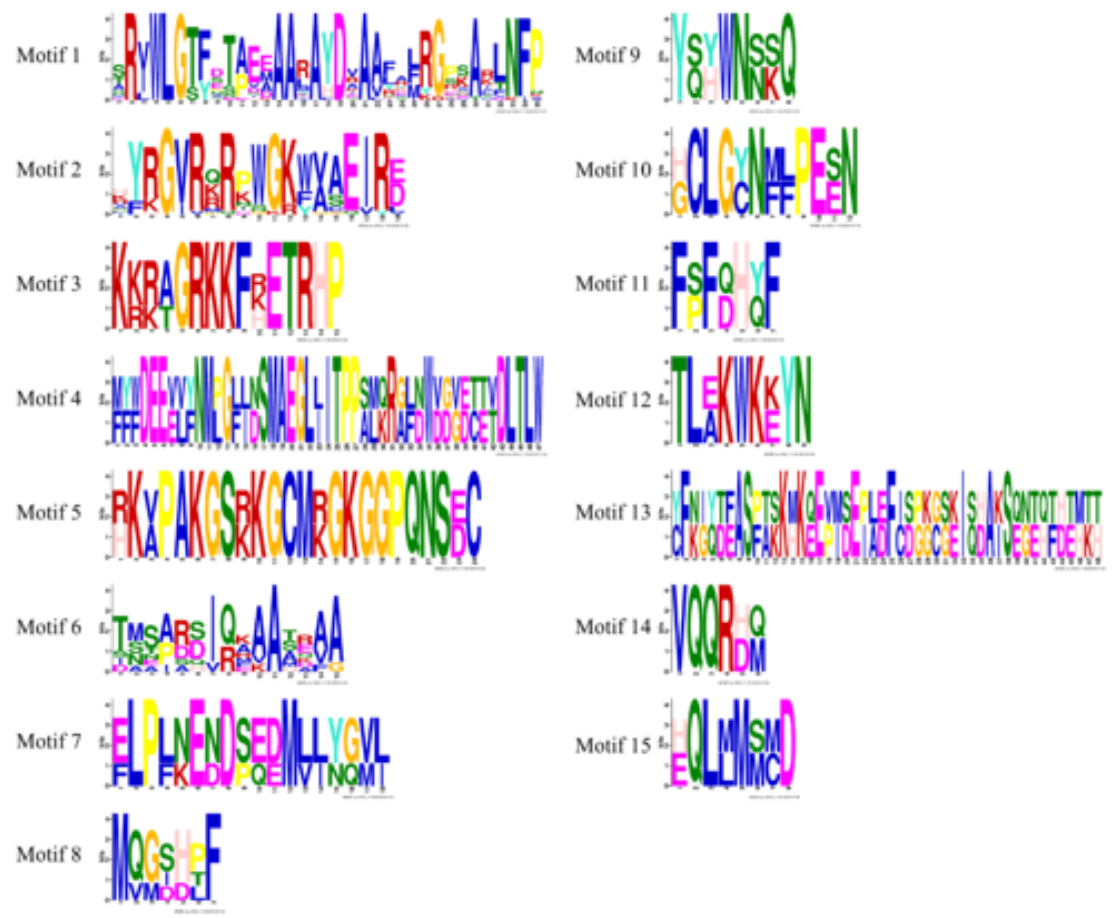

Figure 4

The distribution of the conserved motifs in the AP2/ERF family of transcription factors from C. intermedia a, distribution map of the motifs in the 22 sequences. b, sequences of the conserved motifs. 
Fig. 5

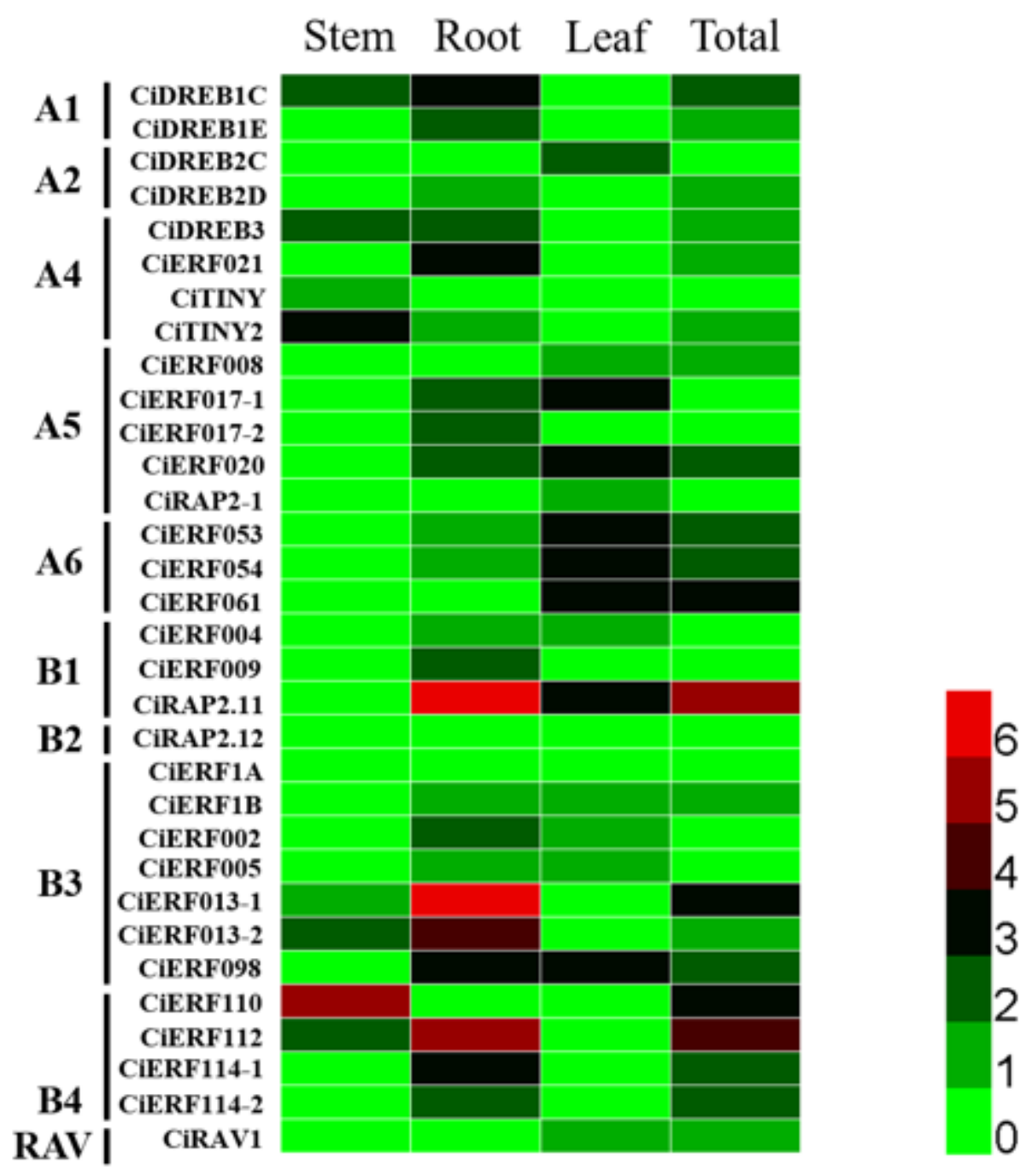

Figure 5

Analysis of the tissue-specific expression patterns of the genes encoding the AP2/ERF family of transcription factors in C. intermedia After LOG2 normalization of the qRT-PCR results, a heat map was plotted using Heml 1.0 software. The different colors indicate different gene expression levels. Specifically, red indicates a high gene expression level, whereas green indicates a low gene expression level. 
Fig. 6
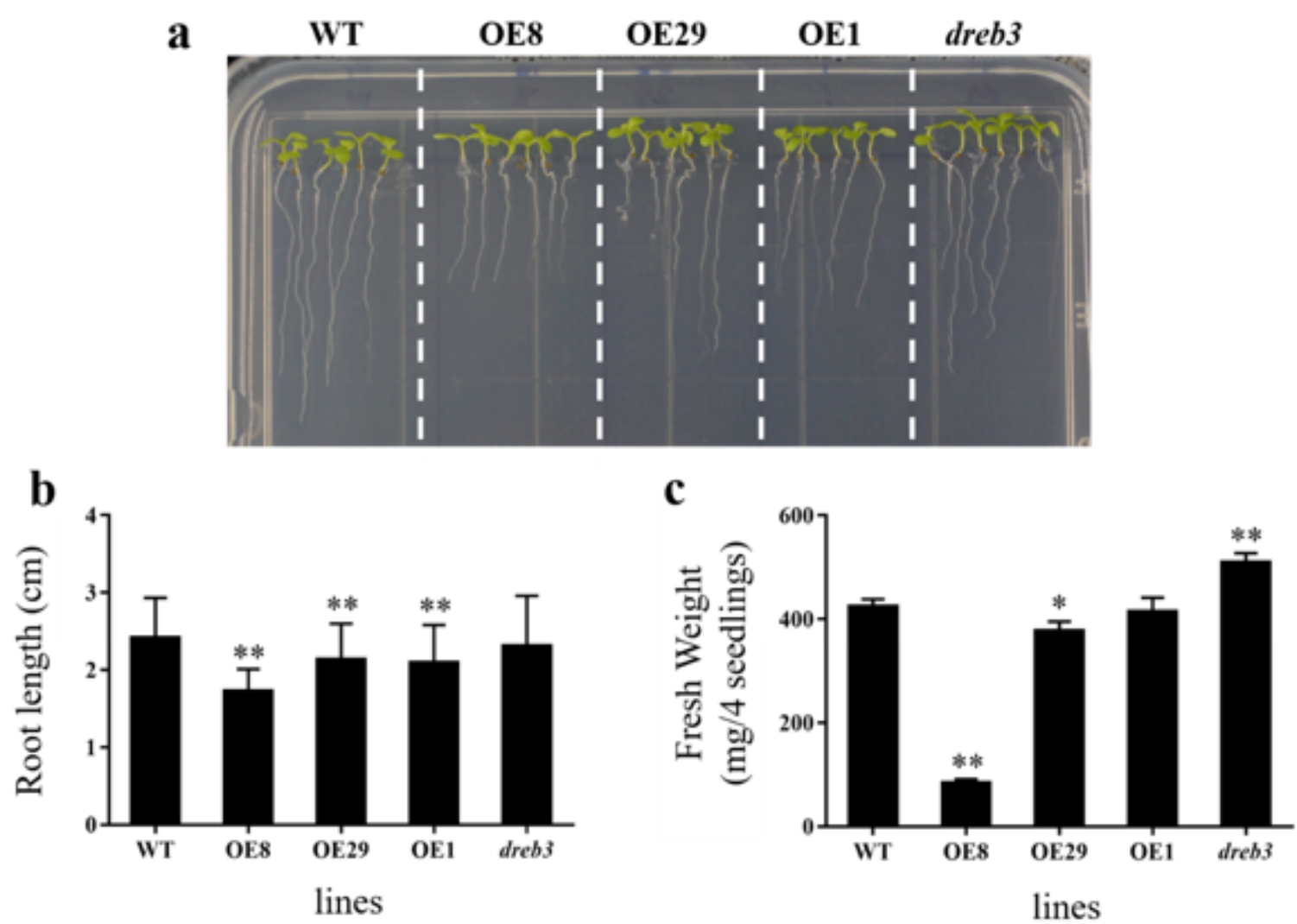

Figure 6

Root length measurement of CiDREB3 transgenic lines, mutant and wild type a: Seedlings grow for 1 weeks; b: Statistics of fresh weight $(n=12)$; : Measurements of root length $(n=40)$; d: gemination rate of day 1 ( $n=165) ;$ OE8, OE29 and OE1 represents transgenic line 8, transgenic line 29 and transgenic lines 1. dreb3 represents mutant (SALK-206788C). Significance level was analyzed using $t$ text. *: Significant difference $(P<0.05) ; * *$ : Extremely significant difference $(P<0.01)$ 


\section{Fig. 7}

a
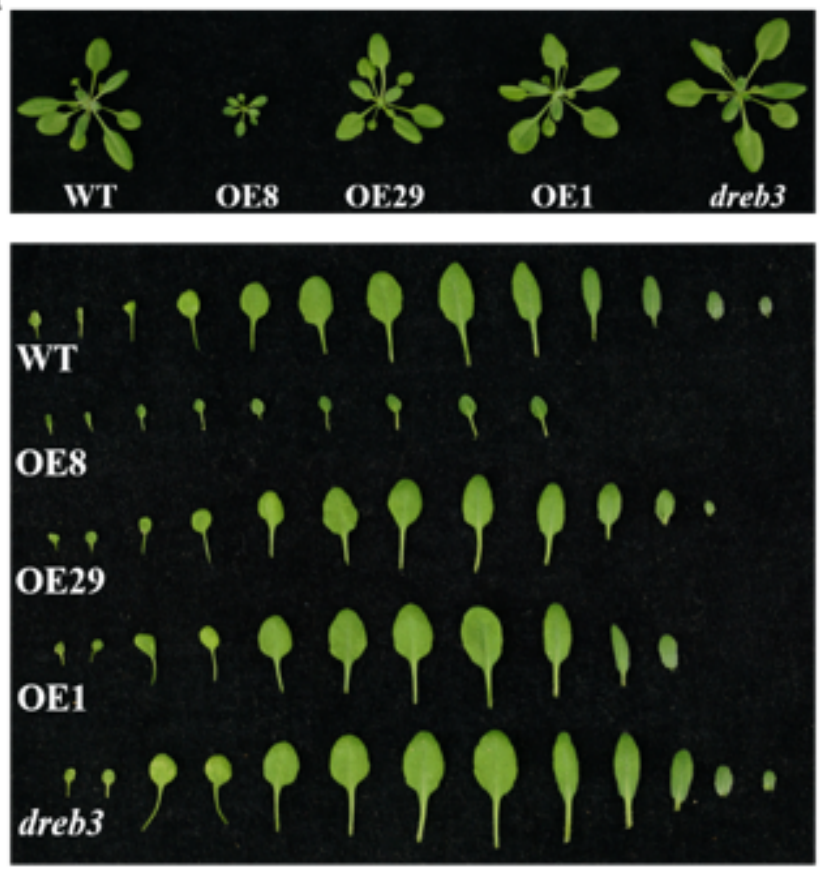

b

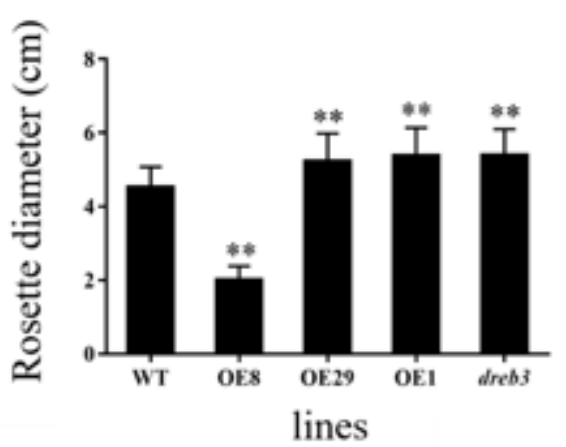

c

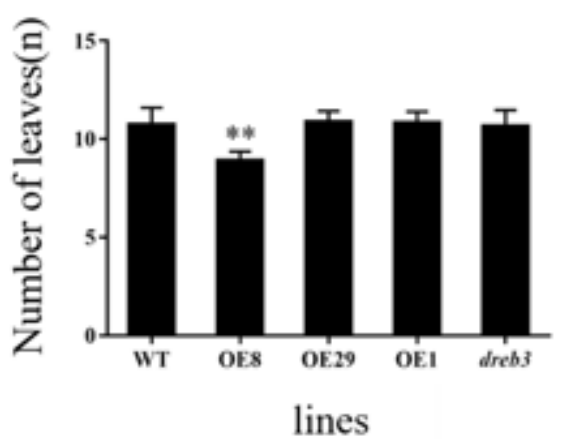

Figure 7

Observation of CiDREB3 transgenic lines, mutant and wild type leaves a: Seedlings grow for 3 weeks; b: Measurements of rosette diameter $(n=30)$; c: Statistics of number of leaves( $n=30)$; OE8, OE29 and OE1 represents transgenic line 8 , transgenic line 29 and transgenic lines 1 . dreb3 represents mutant (SALK206788C). Significance level was analyzed using t text. *: Significant difference $(P<0.05)$; **: Extremely significant difference $(P<0.01)$ 
Fig. 8

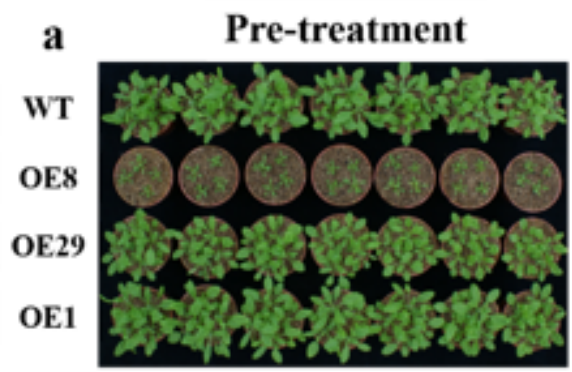

b
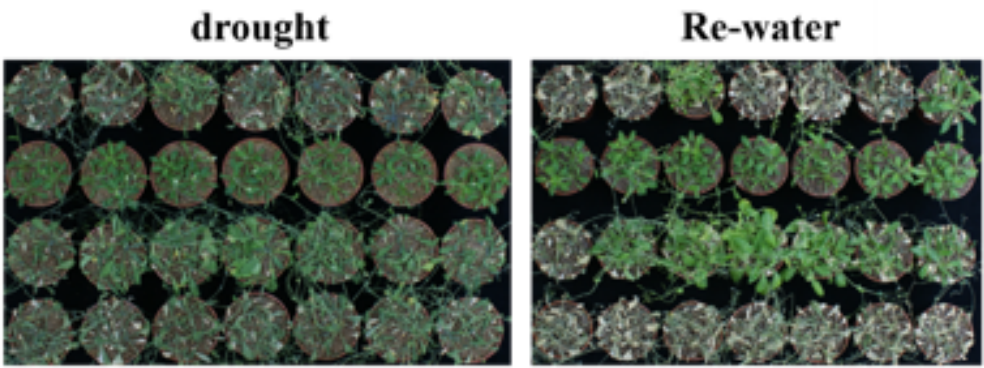

c

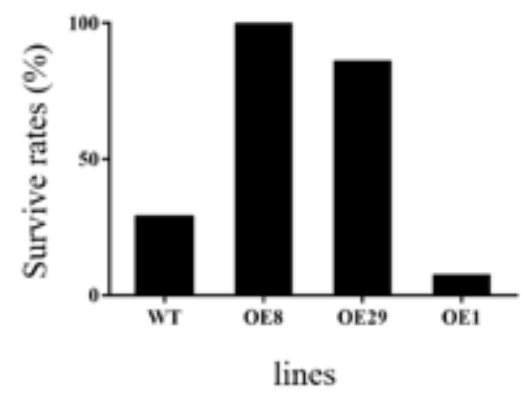

\section{Figure 8}

Phenotypic analysis of CiDREB3 transgenic lines and wild type under drought treatment a: Drought treated seedlings; $b$ : Detection of chlorophyll content; c: Detection of survival rates $(n=40) ; 0 E 8,0 E 29$ and OE1 represents transgenic line 8, transgenic line 29 and transgenic lines 1 . Significance level was analyzed using $t$ text. *: Significant difference $(P<0.05)$; **: Extremely significant difference $(P<0.01)$

\section{Supplementary Files}

This is a list of supplementary files associated with this preprint. Click to download.

- SupplementalFigures.docx

- SupplementalTables1.docx

- Table1.pdf

- Table2.pdf

- Table3.pdf

- Table4.pdf 